\title{
Step Dynamics on Au(110) Studied with a High-Temperature, High-Speed Scanning Tunneling Microscope
}

\author{
L. Kuipers, M. S. Hoogeman, and J. W. M. Frenken \\ Foundation for Fundamental Research on Matter (FOM)-Institute for Atomic and Molecular Physics, \\ Kruislaan 407, 1098 SJ Amsterdam, The Netherlands
}

(Received 12 July 1993)

\begin{abstract}
The dynamics of monoatomic steps on the Au(110) surface was studied with a scanning tunneling microscope from room temperature to $590 \mathrm{~K}$. The time dependence of the position fluctuations of steps was measured as a function of temperature and kink density. The mean-square displacement of the position was found to be proportional to the square root of time. The proportionality constant exhibits Arrhenius behavior and varies linearly with the kink density. The step dynamics is dominated by the diffusion of geometrical kinks that cannot pass each other.
\end{abstract}

PACS numbers: $68.35 . \mathrm{Fx}, 05.40 .+\mathrm{j}, 61.16 . \mathrm{Ch}$

Steps play a major role in many surface phenomena. For instance, they can act as nucleation sites for the growth of new layers and can provide preferred adsorption and reaction sites. The dynamics of steps is of crucial importance for mass transport in growth and erosion phenomena, as well as for surface phase transitions such as surface roughening, deconstruction, and faceting. A scanning tunneling microscope (STM) provides the means to study step dynamics on the atomic scale [1-4]. The atomic mechanism underlying the thermal movements of steps is not yet fully understood. Up to now, the STM has been used to investigate the so-called "frizziness" of steps on metal surfaces, for instance on $\mathrm{Cu}(001)$ and $\mathrm{Ag}(111)$ [1-3]. Frizziness is the phenomenon that a step appears rough in the STM due to an undersampling of the step in time. The results on the frizzy steps have been interpreted in terms of the thermal creation of kink pairs. In this scenario a pair of kinks of opposite direction is formed when one or more atoms either depart from or attach to a previously straight section of the step.

In this Letter, we present a direct observation and a temperature-dependent statistical analysis of step dynamics on $\mathrm{Au}(110)$ performed with a high-speed, hightemperature STM. The individual snapshot observations show that the step fluctuates due to the diffusion of preexisting kinks along the step and that thermal kink generation plays no role of importance in the step dynamics at the investigated temperatures. The dependence of the mean-square displacement of the step on time and kink density indicates that the kinks move due to the exchange of atoms between kink sites and adatom sites on the adjacent terraces. From the temperature dependence we derive the activation energy for the movement of a single kink.

The experiments were performed in ultrahigh vacuum $\left(p<1 \times 10^{-10}\right.$ mbar) with a STM specially designed for use at high temperatures. This instrument has been used to image various metal and semiconductor surfaces with atomic resolution up to $750 \mathrm{~K}$. The STM tip was prepared by electrochemical etching of a $0.25 \mathrm{~mm}$ diame- ter $\mathrm{W}$ wire and annealing in vacuum. The tip was further prepared in situ by field electron emission and $\mathrm{Ar}$ ion sputtering. The Au sample was chemically etched and mechanically polished. It was cleaned in situ by cycles of Ar ion sputtering and annealing to $550 \mathrm{~K}$. The cycles were optimized to produce a sharp $(1 \times 2)$ low-energy electron diffraction pattern with a low background intensity. During the initial stages of sample preparation we found with Auger-electron spectroscopy (AES) that the surface was contaminated with $\mathrm{Ca}$, which segregated from the bulk to the surface. After several tens of cleaning cycles the level of impurities was below the $1 \%$ detection limit of AES. By radiative heating of the rear side of the crystal, temperatures up to $590 \mathrm{~K}$ were obtained. The temperature was monitored with an infrared pyrometer (Ircon model 6000) and a chromel-alumel thermocouple connected directly to the sample.

All STM data presented in this Letter were obtained from measurements with the same tunneling current of $0.1 \mathrm{nA}$ and bias voltages in the range of -0.2 to $-0.9 \mathrm{~V}$. We have found no dependence of the step dynamics on the applied bias voltage.

Figure 1 shows a sequence of four surface topographs of $\mathrm{Au}(110)$, measured at $374 \mathrm{~K}$, at a rate of 1 image every $49 \mathrm{~s}$. The images show an island of monoatomic height on top of a terrace. The $(1 \times 2)$ missing-row reconstruction of the surface is clearly present on both the terrace and the island. Both steps in Figs. 1(a)-1(d) are of the chiral, i.e., (111), type. All the step dynamics discussed in this study concerns this low-energy type of step. The steps on either side of the island contain kinks, and the sequence of images in Fig. 1 shows the mobility of the kinks at this temperature. Each movement of a kink causes the position of the step to locally change by one unit of the missing-row reconstruction. If the sampling rate of the step position is slow compared to the mobility of the kinks, this causes the occurrence of apparent kink pairs. Arrows A in Fig. 1(d) indicate such an event, where it appears as if the step contains two nearby kinks of opposite direction. Each time that a kink crosses the 

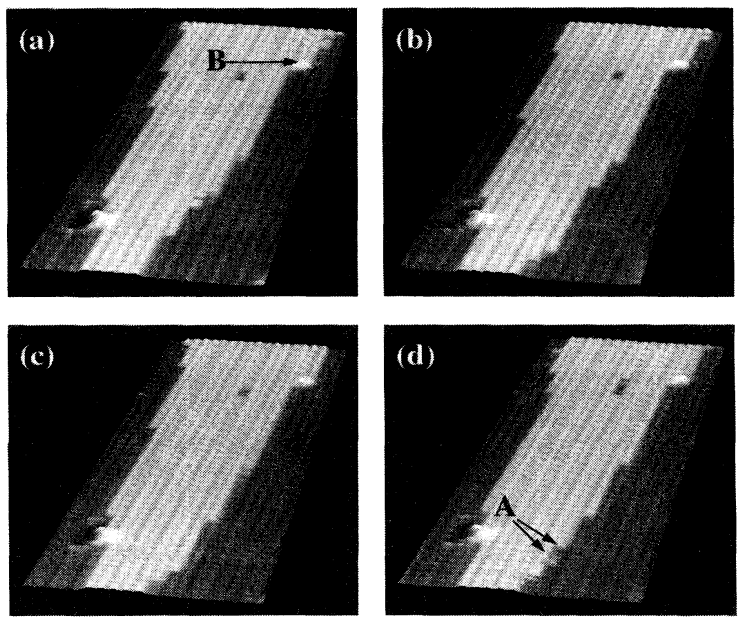

FIG. 1. Sequence of four STM images of the Au(110) surface $\left(160 \AA \times 513 \AA, V_{t}=-0.88 \mathrm{~V}, I_{t}=0.1 \mathrm{nA}\right)$ measured at $375 \mathrm{~K}$ at a rate of 1 image $/ 49 \mathrm{~s}$.

line being scanned, the image shows a jump in the position of the step. Multiple crossings of the kink through the scan line lead to a typical telegraph noise of the step location. These features have been observed on several metal surfaces, even at room temperature [1-3, 5-8], and have been termed "frizzes" [1]. We observe that the frizziness decreases or even disappears completely when the line rate of the measurement is increased sufficiently. At high scan speeds and/or low temperatures (see Fig. 1) we observe that the step dynamics occurs only by diffusion of preexisting kinks in the steps. The kinks seem to diffuse freely, but they do not pass each other, thus avoiding "overhangs" in the step shape. We are forced to conclude that the thermal creation of kink pairs can only play a minor role in the step dynamics on $\mathrm{Au}(110)$. This is in contrast with the conclusions for $\mathrm{Cu}(001)$ and $\operatorname{Ag}(1111)$ in Refs. [1,2].

The STM images further reveal that all steps on the surface are pinned. Arrow B in Fig. 1(a) indicates the most common pinning center, which appears as an immobile protrusion at a step. We assume that it consists of one or more segregated $\mathrm{Ca}$ atoms. The typical density of these protrusions is between $10^{-4}$ and $10^{-3}$ monolayer, and the average distance between protrusions along a step is $400 \AA$. If a step is not pinned precisely parallel to the close-packed [1 $1 \overline{1} 0$ ] surface azimuth, this local misorientation has to be accommodated via geometrically enforced kinks. The local kink density is then the ratio of the number of these geometrical kinks and the distance between the two pinning centers enclosing them. At all temperatures in this study the pinning centers were immobile. From the fact that the step dynamics at each temperature depended only on the kink density and not on the distance between the pinning centers, we conclude that the pinning centers did not affect the dynamics of the

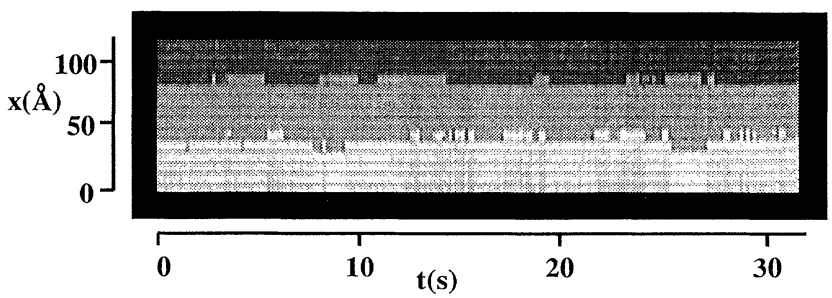

FIG. 2. Time sequence of a line across two steps, measured at $475 \mathrm{~K}\left(32 \mathrm{~s} \times 118 \AA, V_{t}=-0.60 \mathrm{~V}, I_{t}=0.1 \mathrm{nA}\right)$. The time per scan line is $83 \mathrm{~ms}$.

enclosed section of the steps. This enabled us to investigate the step dynamics as a function of kink density, by simply selecting step sections pinned along different orientations. When the step was pinned perfectly along the $[1 \overline{1} 0]$ direction, the step position between the pinning centers did not move over large time intervals, again demonstrating that thermal kink creation at these temperatures is negligible.

We quantified the step mobility by measuring the step position fluctuations in time, at a point midway between the two pinning centers. This was done by repeatedly scanning the same line along a direction perpendicular to the step. A typical measurement of step positions as a function of time is depicted in Fig. 2 for two neighboring steps. The vertical axis denotes the lateral coordinate perpendicular to the steps, and the horizontal axis corresponds to time. Figure 2 demonstrates that the position of a step could easily be determined with missing-row resolution.

From the measured time dependence of the step position $x(t)$, we calculate the mean-square displacement $\sigma_{x}^{2}(t)=\left\langle\left[x\left(t+t_{0}\right)-x\left(t_{0}\right)\right]^{2}\right\rangle$, where the angular brackets denote an average over all times $t_{0}$. Figure 3 shows a double-logarithmic representation of a typical correlation function, for a step with an average distance between kinks of 64.3 sites, measured at $556 \mathrm{~K}$. The inset shows the same data on a linear scale. Clearly, $\sigma_{x}^{2}(t)$ obeys a power law. We find that the power is $0.48 \pm 0.05$ for all kink densities and temperatures. We can therefore write $\sigma_{x}^{2}(t)=m(N, T) t^{0.48 \pm 0.05}$, where the prefactor $m$, which is a measure of the step mobility, may depend on the average number of lattice sites between kinks $N$ and the temperature $T$.

Figure 4 shows the dependence of $m(N, T)$ on $N$ for different temperatures, which is described well by $m(N$, $T)=c(T) N^{-0.96 \pm 0.12}$, where $c(T)$ contains the temperature dependence of the step mobility. In order to obtain the activation energy for the movement of a single kink, we fit $m(N, T)$ for each temperature with $c(T) N^{-1}$. Figure 5 shows an Arrhenius plot of $c(T)$. The data fall on a straight line, indicating that the step motion is a thermally activated process. The slope of the line corresponds to an activation energy of $0.7 \pm 0.1 \mathrm{eV}$ (see below). 


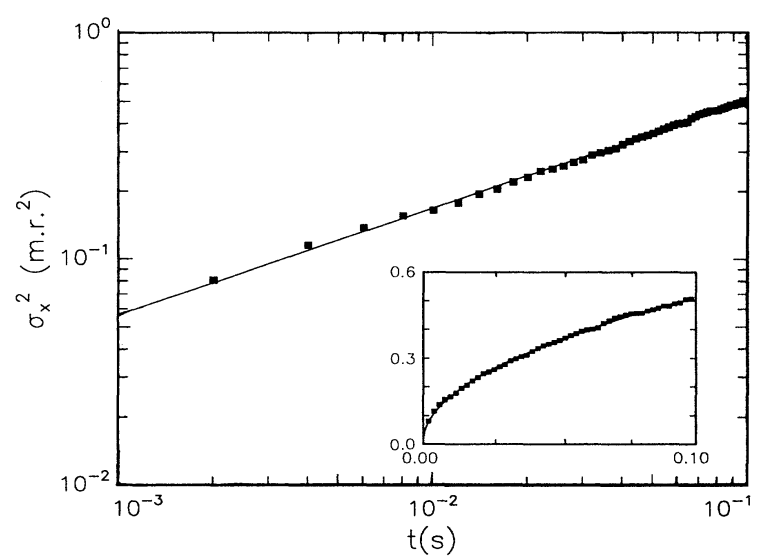

FIG. 3. Log-log plot of $\sigma_{x}^{2}(t)$ of a step with an average distance between kinks of 64.3 lattice sites, at a temperature of $556 \mathrm{~K}$. The mean-square displacements have been expressed in units of the square of the missing-row spacing. The inset shows $\sigma_{x}^{2}(t)$ in a linear plot. The solid curves are power-law fits, with $\sigma_{x}^{2}(t) \propto t^{1 / 2}$

Based on the observation at low temperature (Fig. 1) that the step motion is brought about by the diffusion of geometrical kinks, we now try to model the dependence of the step wandering on time, kink density, and temperature. At any observation point along the step, the step displacement $\Delta x(t)$, after time $t$, reflects the difference between the number of kinks that have passed the point from left to right and the number of kinks that have passed in the opposite direction. If the kinks perform a random walk and their motion is uncorrelated, the probability density for $K$ kinks to pass from left to right is the Gaussian $(1 / 2 \pi v t)^{1 / 2} \exp \left[-(K N)^{2} / 2 v t\right]$, where $v=v_{0}$ $\times \exp \left[-E_{\mathrm{act}} / k_{B} T\right]$ is the kink displacement frequency. The mean-square step displacement $\sigma_{x}^{2}(t)$, in a direction perpendicular to the kink motion, is obtained from the average number of kinks passing the observation point in one direction:

$\sigma_{x}^{2}(t)=\frac{2}{N \sqrt{2 \pi v t}} \int_{0}^{\infty} y e^{-y^{2} / 2 v t} d y=\frac{1}{N}\left[\frac{2}{\pi} v t\right]^{1 / 2}$.

The time exponent of $\frac{1}{2}$, which should also apply to the mean-square displacement for long times of the individual kinks, forms a general result for one-dimensional diffusion of nonpassing objects (see, e.g., [9]). The time exponent of $\frac{1}{2}$ further indicates that the kinks move by exchanging atoms with a lattice gas of adatoms on the adjacent terraces. When there is no exchange of kink atoms with a lattice gas of atoms on the terraces, for example, when kinks would only move by direct exchange of atoms with neighboring kinks, the long-time dynamics of both steps and kinks should slow down to a time exponent of $\frac{1}{4}[10]$.

Equation (1) describes both the time dependence of $\sigma_{x}^{2}$ (Fig. 3) and its dependence on $N$ and $T$ (Figs. 4 and 5).

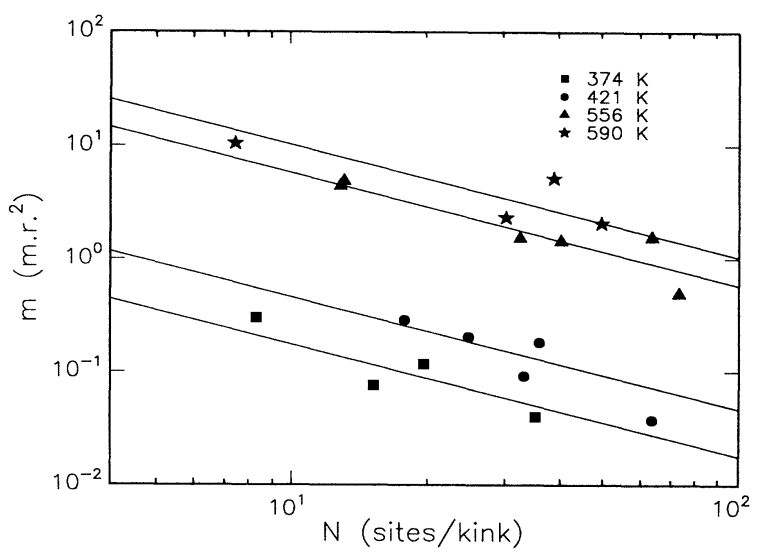

FIG. 4. Log-log plot of the mean-square-displacement prefactor $m(N, T)$ versus average kink distance $N$ for different temperatures. The prefactor $m(N, T)$ has been expressed in units of the square of the missing-row spacing. The solid lines are fits with $m(N, T)=c(T) N^{-1}$.

Note that the activation energy $E_{\text {act }}$ of $0.7 \mathrm{eV}$ is twice the slope of the straight line in Fig. 5 [see Eq. (1)]. Using Eq. (1), we calculate $v_{0}$ to be $10^{10 \pm 1} \mathrm{~Hz} . E_{\text {act }}$ is the activation energy associated with the displacement of a kink over one lattice site. Because of the reconstruction of $\mathrm{Au}(110)$, such a displacement involves the exchange of two atoms with the terrace, one in the first atomic layer and one in the second. This might account for the low value of $v_{0}$ compared to typical vibration frequencies. We are currently calculating activation energies based on the effective-medium theory [11].

The role of nonthermal kinks has also been suggested by a recent study [3] of frizziness on two vicinal surfaces of $\mathrm{Cu}(001)$. The $(1 \times 2)$ reconstruction of $\mathrm{Au}(110)$ probably causes a subtle difference in dynamics with that on $\mathrm{Cu}(001)$. For a nonreconstructed surface such as

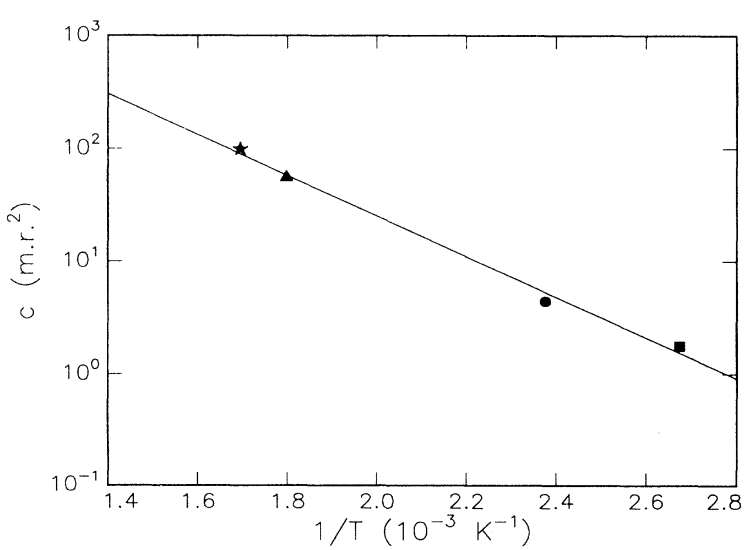

FIG. 5. Arrhenius plot of the prefactor $c(T)$, expressed in units of the square of the missing-row spacing. The solid line is a fit according to $c(T) \propto \exp \left(E_{\text {act }} / 2 k_{B} T\right)$, with $E_{\text {act }}=0.72 \mathrm{eV}$. 
$\mathrm{Cu}(001)$, it seems likely that after having detached from a kink each adatom would perform a random walk along the step, and finally stick either at the next kink or at the original one. Within this scenario, the probability for a newly evaporated adatom to reach the next kink before revisiting the original one would be $N^{-1}$. This effectively causes the frequency with which the position of the kink successfully changes to be reduced by a factor $N^{-1}$. As explained above, the direct exchange of atoms between neighboring kinks would lead to a time exponent of $\frac{1}{4}$. We thus expect $\sigma_{x}^{2}(t) \propto N^{-3 / 2} t^{1 / 4}$ for surfaces such as $\mathrm{Cu}(001)$. The fact that we observe $\sigma_{x}^{2}(t) \propto N^{-1} t^{1 / 2}$ instead on $\mathrm{Au}(110)$ is direct evidence for kink movement via exchange of atoms with adjacent terraces. We suggest that the missing-row troughs on Au(110) effectively shield the adatoms from the neighboring kinks [12].

The observations presented in this Letter demonstrate that the step pinning by impurities can strongly increase the kink density with respect to the thermally generated concentration of kinks. Thus, while the pinning immobilizes the steps on large length scales it makes them extremely dynamic on length scales below the average distance between pinning sites. Since defects and impurities can never be avoided completely, one should expect this general conclusion to apply also to crystal surfaces other than $\mathrm{Au}(110)$. Finally, we propose that adsorbates that increase the kink density via step pinning could act as efficient promoters for catalytic surface reactions which involve kink sites.

The authors gratefully acknowledge stimulating discussions with $\mathrm{H}$. van Beijeren, B. Mulder, and M. den Nijs.
We thank R. J. I. M. Koper for the preparation of the Au sample and J. S. Custer for a critical reading of the manuscript. This work is part of the research program of the Foundation for Fundamental Research on Matter (FOM) and was made possible by financial support from the Netherlands Organization for Scientific Research (NWO).

[1] M. Poensgen, J. F. Wolf, J. Frohn, M. Giesen, and H. Ibach, Surf. Sci. 274, 430 (1992).

[2] M. Giesen, J. Frohn, M. Poensgen, J. F. Wolf, and H. Ibach, J. Vac. Sci. Technol. A 10, 2597 (1992).

[3] J. C. Girard, S. Gauthier, S. Rousset, W. Sacks, S. de Cheveigné, and J. Klein (private communication).

[4] N. Kitamura, B. S. Swartzentruber, M. G. Lagally, and M. B. Webb, Phys. Rev. B 48, 5704 (1993).

[5] Y. Kuk, F. M. Chua, P. J. Silverman, and A. J. Meyer, Phys. Rev. B 41, 12393 (1990).

[6] J. Winterlin, R. Schuster, D. J. Coulman, G. Ertl, and R. J. Behm, J. Vac. Sci. Technol. B 10, 902 (1991).

[7] S. Rousset, S. Chiang, D. E. Fowler, and D. D. Chambliss, Phys. Rev. Lett. 69, 3200 (1992).

[8] L. Kuipers and J. W. M. Frenken, Phys. Rev. Lett. 70, 3907 (1993).

[9] H. van Beijeren, K. W. Kehr, and R. Kutner, Phys. Rev. B 28, 5711 (1983), and references therein.

[10] N. C. Bartelt, J. L. Goldberg, T. L. Einstein, and E. D. Williams, Surf. Sci. 273, 252 (1992).

[11] L. Kuipers, M. S. Hoogeman, and J. W. M. Frenken (to be published).

[12] H. van Beijeren (private communication). 

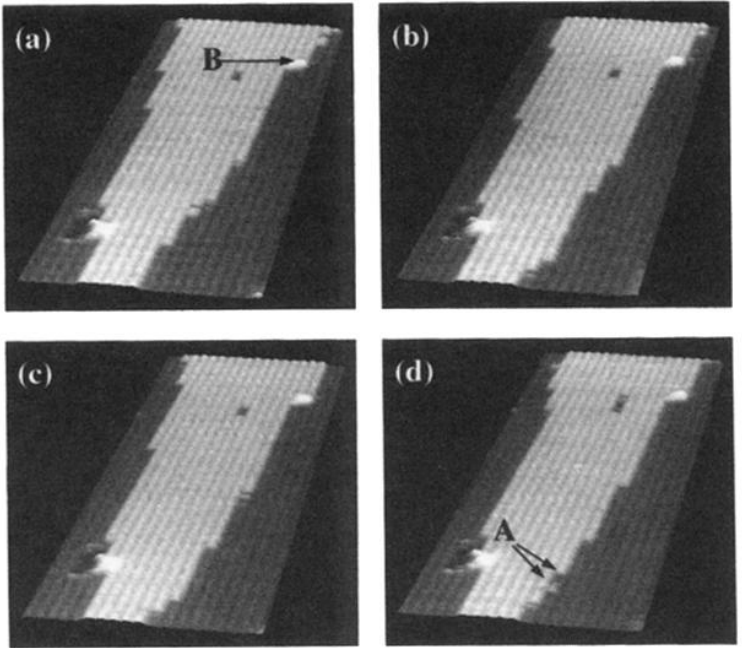

FIG. 1. Sequence of four STM images of the Au(110) surface $\left(160 \AA \times 513 \AA, V_{t}=-0.88 \mathrm{~V}, I_{t}=0.1 \mathrm{nA}\right)$ measured at $375 \mathrm{~K}$ at a rate of 1 image/49 s. 


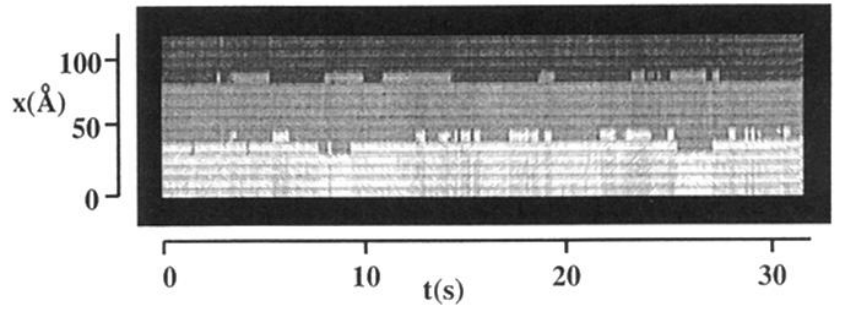

FIG. 2. Time sequence of a line across two steps, measured at $475 \mathrm{~K}\left(32 \mathrm{~s} \times 118 \AA, V_{t}=-0.60 \mathrm{~V}, I_{t}=0.1 \mathrm{nA}\right)$. The time per scan line is $83 \mathrm{~ms}$. 